\title{
BMJ Global Health A long way to go: a systematic review to assess the utilisation of sexual and reproductive health services during humanitarian crises
}

\author{
Neha S Singh, ${ }^{1,2}$ Sarindi Aryasinghe, ${ }^{2}$ James Smith, ${ }^{1}$ Rajat Khosla, ${ }^{3}$ Lale Say, ${ }^{3}$ \\ Karl Blanchet ${ }^{1}$
}

\begin{abstract}
To cite: Singh NS, Aryasinghe S Smith J, et al. A long way to go: a systematic review to assess the utilisation of sexual and reproductive health services during humanitarian crises. BMJ Glob Health 2018:3:e000682. doi:10.1136/ bmjgh-2017-000682
\end{abstract}

Handling editor Soumitra Bhuyan

- Additional material is published online only. To view, please visit the journal online (http://dx.doi.org/10.1136/ bmjgh-2017-000682).

Received 14 December 2017 Revised 12 February 2018 Accepted 2 March 2018
Check for updates

${ }^{1}$ Health in Humanitarian Crises Centre, London School of Hygiene and Tropical Medicine, London, UK

${ }^{2}$ Centre for Maternal, Adolescent, Reproductive and Child Health (MARCH), London School of Hygiene and Tropical Medicine, London, UK ${ }^{3}$ Department of Reproductive Health and Research, World Health Organization, Geneva, Switzerland

Correspondence to Dr Neha S Singh;

neha.singh@|shtm.ac.uk

\section{ABSTRACT}

Introduction Women and girls are affected significantly in both sudden and slow-onset emergencies, and face multiple sexual and reproductive health (SRH) challenges in humanitarian crises contexts. There are an estimated 26 million women and girls of reproductive age living in humanitarian crises settings, all of whom need access to SRH information and services. This systematic review aimed to assess the utilisation of services of SRH interventions from the onset of emergencies in low- and middle-income countries.

Methods We searched for both quantitative and qualitative studies in peer-reviewed journals across the following four databases: EMBASE, Global Health, MEDLINE and PsychINFO from 1 January 1980 to 10 April 2017. Primary outcomes of interest included selfreported use and/or confirmed use of the Minimum Initial Service Package services and abortion services. Two authors independently extracted and analysed data from published papers on the effect of SRH interventions on a range of SRH care utilisation outcomes from the onset of emergencies, and used a narrative synthesis approach. Results Of the 2404 identified citations, 23 studies met the inclusion criteria. $52.1 \%$ of the studies $(n=12)$ used quasi-experimental study designs, which provided some statistical measure of difference between intervention and outcome. $39.1 \%$ of the studies $(n=9)$ selected were graded as high quality, $39.1 \%$ moderate quality $(n=9)$ and $17.4 \%$ low quality $(n=4)$. Evidence of effectiveness in increasing service utilisation was available for the following interventions: peer-led and interpersonal education and mass media campaigns, community-based programming and three-tiered network of community-based reproductive and maternal health providers.

Conclusions Despite increased attention to SRH service provision in humanitarian crises settings, the evidence base is still very limited. More implementation research is required to identify interventions to increase utilisation of SRH services in diverse humanitarian crises settings and populations.

\section{INTRODUCTION}

Sexual and reproductive health (SRH) and rights are fundamental to individual health

\section{Key questions}

What is already known?

- There are an estimated 32 million women and girls of reproductive age living in humanitarian crises settings, all of whom need sexual and reproductive health (SRH) information and services.

- Previous studies yielded mixed evidence for the implementation of SRH services in humanitarian crises.

- There has been no peer-reviewed study since 2004 that has comprehensively evaluated all pillars of the Minimum Initial Service Package for SRH in humanitarian crises settings.

What are the new findings?

- Interpersonal and peer-led education and mass media campaigns are effective interventions for increasing service utilisation of HIV, sexually transmitted infection and maternal health services in crises settings.

- Training lower cadre health workers from refugee or internally displaced populations to provide SRH services in crises settings can be effective in increasing SRH service use.

What do the new findings imply?

- The limited evidence base for SRH interventions highlights the need for improved research on the utilisation of SRH interventions in humanitarian crises.

- Rigorous implementation research is needed across a range of diverse crisis settings and populations to identify evidence-based interventions to increase the utilisation of SRH services.

and well-being, as well as population health and development. Significant gaps in access to SRH information and services exist globally, which threaten the lives and well-being of individuals and their families. Unmet need for SRH information and services is highest among the most vulnerable-adolescents, populations with low socioeconomic status, 
those living in rural areas and urban slums, people living with HIV, internally displaced people and those living in humanitarian crises contexts. ${ }^{1}$ We define humanitarian crises as a significant disruption of the functioning of a community or society causing widespread human, material, economic or environmental losses, which exceed the ability of those affected to cope using its own resources, necessitating a request to the national or international level for external assistance. The crisis situation may be man-made (eg, armed conflict) or natural (eg, drought). Individuals living in humanitarian crises face significant barriers and challenges, which impede their access to healthcare more generally and sexual and reproductive healthcare in particular. ${ }^{1}$ These challenges are particularly exacerbated for women and girls.

Women and girls are affected significantly in both sudden and slow-onset humanitarian crises, and face multiple SRH challenges in these contexts-there are an estimated 32 million women and girls of reproductive age (ie, 15-49 years) living in humanitarian crises situations, all of whom need SRH information and services. ${ }^{2}$ Humanitarian crises can increase the risk of poor SRH outcomes due to reduced access to services and supplies, damaged health facilities and increased exposure to sexual violence among other factors, and have implications across the life cycle, eg, increased risk of maternal and newborn morbidity and mortality.

In order to address this need, the Inter-Agency Working Group on Reproductive Health in Crisis (IAWG), a consortium of non-governmental organisations, donors, governments and the United Nations agencies, developed a technical and programmatic guide, Reproductive Health in Refugee Situations: An Inter-Agency Field Manual, to provide guidance to field staff on reproductive health interventions during emergencies. ${ }^{3}$ Specifically, the Field Manual includes a chapter on the Minimum Initial Service Package (MISP), to be implemented at the onset of every humanitarian emergency, via the following four programme pillars: (i) prevent and manage the consequences of sexual violence; (ii) reduce HIV transmission; (iii) prevent maternal and newborn death and illness and (iv) plan for comprehensive sexual and reproductive health care, integrated into primary healthcare, as the humanitarian crises situation permits.

While some progress has been made with regard to the availability of SRH services in humanitarian crises (eg, funding for certain services), significant challenges remain with regard to utilisation of these services. These challenges are further compounded by overall gaps in health systems capacity. Recent systematic reviews have found that the absence of quality data on women's, children's and adolescents' health in emergencies hinders design and implementation of sustainable interventions. ${ }^{14}$ Another systematic review by Warren et al. highlighted evidence gaps in the effectiveness of delivering and scaling-up public health interventions in humanitarian crises, as well as in evidence for populations using SRH services. ${ }^{5}$ It also indicated that the MISP was not being systematically implemented, and that core SRH services were being neglected (eg abortion, contraception, care for adolescents). Failure to implement the essential SRH services as an integrated package has significant consequences for individuals in humanitarian crises settings. Furthermore, the population of displaced individuals has increased in recent decades, and increased resources are needed to improve health outcomes. ${ }^{4}$ A recent systematic review noted the dearth of studies measuring SRH outcomes in humanitarian crises $^{4}$; however, a key challenge in improving utilisation of quality SRH services has also been a lack of evidence, and there has been no systematic review to date that has assessed the utilisation of these services in crises settings. Data are needed to inform how to most effectively increase utilisation of services, and to advocate for the SRH needs of individuals living in humanitarian crises.

To build on progress made, we aimed to consolidate existing evidence for the utilisation of SRH interventions, including the MISP, from the onset of emergencies by conducting a systematic review. This study aimed to assess the utilisation of SRH services in humanitarian crises in low- and middle-income countries from 1980 to 2017. We also aimed to assess issues related to access of SRH interventions including the MISP, eg, whether these services are being used by vulnerable populations such as people with disabilities, sex workers, adolescent girls and lesbian, gay, bisexual, transsexual, queer and intersex populations.

\section{METHODS}

This systematic literature review adheres to the Preferred Reporting Items for Systematic Reviews and Meta-Analyses statement. ${ }^{6}$ It is registered with the PROSPERO database with identifier number CRD42017082191.

\section{Search strategy and selection criteria}

Search terms for SRH were based on the standardised definition from the International Conference on Population and Development in 1994 and the WHO Reproductive Health Strategy, ${ }^{78}$ as well as a previous systematic review by Warren et al. on SRH interventions in humanitarian crises settings. ${ }^{5}$ Our search encompassed terms used in international guidelines on reproductive health in conflict-affected situations, including family planning, abortion, HIV/AIDS and sexually transmitted infections (STIs), prevention of mother-to-child transmission (PMTCT), maternal and newborn health and sexual and gender-based violence. Although humanitarian crises can affect any country, only studies from low-income or middle-income countries were included in this study, as the majority of humanitarian crises occur in these countries, and the resources available to address them are different in high-income countries.

We searched literature from 1 January 1980 to 10 April 2017 as a recent literature review on public health interventions in humanitarian crises yielded no SRH studies 

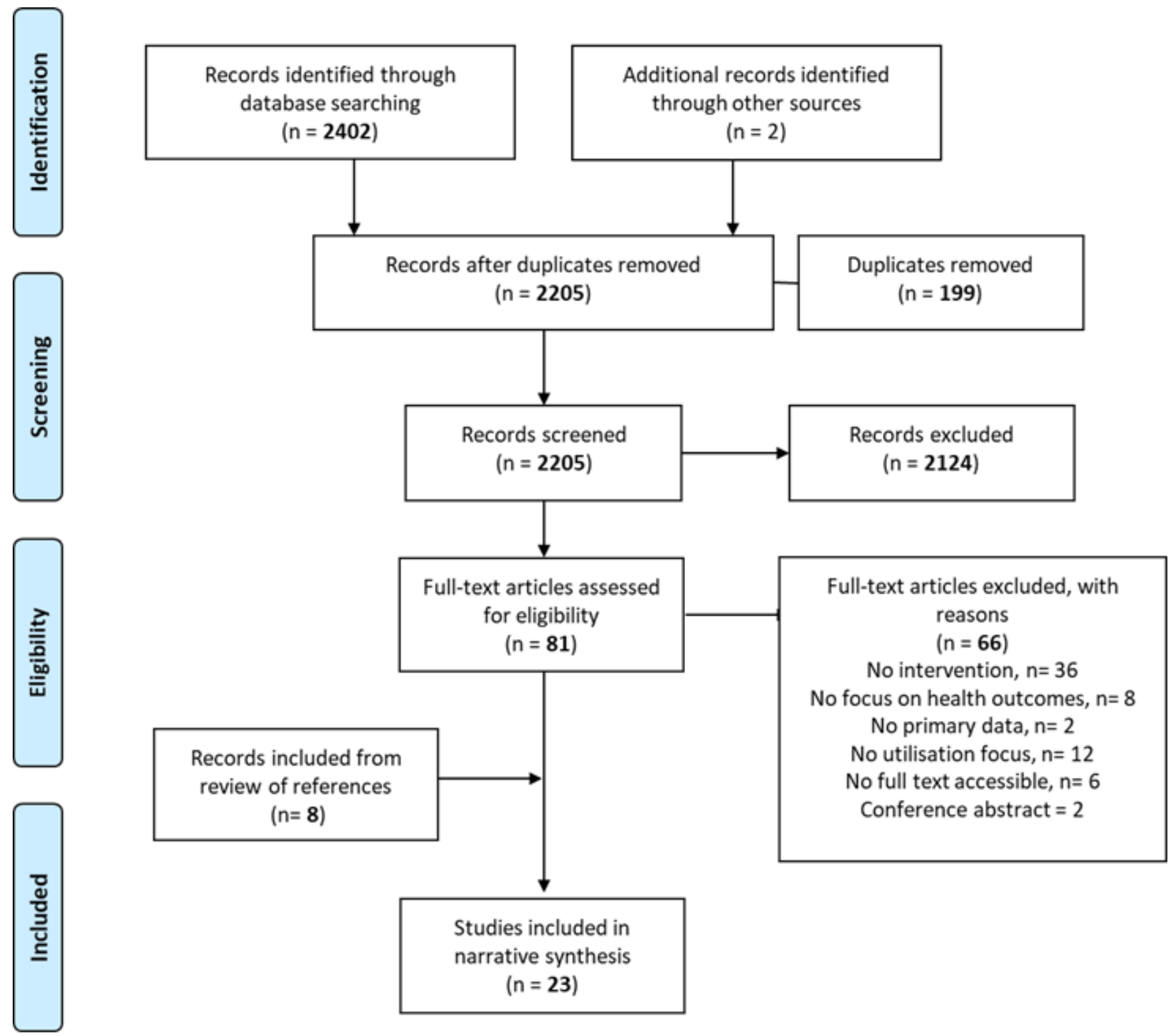

Figure 1 Preferred Reporting Items for Systematic Reviews and Meta-Analyses (PRISMA) flow chart: selection process for systematic review on the utilisation of sexual and reproductive health interventions in humanitarian crises settings.

published prior to $1980 .{ }^{4}$ A detailed protocol with specific search terms and strategy is provided in online supplementary appendix A. This was generated by the authors and expert review, and supplemented by searching for other search strategies used in previous systematic reviews on similar topics. ${ }^{145}$ We also consulted a trained information science and Cochrane review specialist to review our literature searching syntax and strategy. The search strategy followed the following formula: (humanitarian crises-related terms) and (SRH domain, eg contraception-related terms) and (utilisation-related search terms) and (study type-related terms) and (year range) and (low- and middle-income country-related terms).

We included quantitative and qualitative studies from peer-reviewed journals across the following four databases: EMBASE, Global Health, MEDLINE and PsychINFO. We complemented searches by screening the reference lists of (1) papers for potentially relevant studies and (2) relevant systematic reviews. We also consulted experts on SRH service delivery and research to identify research not found during our systematic search.

\section{Data analysis}

We downloaded all returned citations from the searched databases into an Endnote library and applied a standard data-screening process (figure 1). Primary and secondary outcomes and primary outputs of interest for inclusion were based on those from the IAWG field manual. ${ }^{3}$ This is an established and widely used manual for SRH in crisis settings, and was selected based on discussion with an SRH expert committee. Inclusion and exclusion criteria applied during the screening process are outlined in table 1. Data from the final selected studies were extracted into an Excel database, with data 
Table 1 Inclusion and exclusion criteria

\begin{tabular}{lll}
\hline Category & Included & Excluded \\
\hline Population of interest & $\begin{array}{l}\text { Crisis-affected populations receiving humanitarian } \\
\text { assistance or aid in low-income or middle-income } \\
\text { countries (as defined by World Bank, 2012): including } \\
\text { refugees and internally displaced persons (see online } \\
\text { supplementary appendix A for definitions of these } \\
\text { terms) }\end{array}$
\end{tabular}

Intervention Any health-related intervention seeking to improve sexual and reproductive health $(\mathrm{SRH})$ outcomes

Outcomes of interest Primary quantitative research studies: indicators included self-reported use and/or confirmed use of the Minimum Initial Service Package (MISP) services (ie, use of emergency obstetric and newborn care services, use of condoms and/or contraceptives, use of HIV and sexually transmitted infection screening services, use of abortion services, use of adolescent health services and use of psychological support services by sexual violence survivors). Self-reported use is defined as when a beneficiary reports the use of a product or service without additional verification; this indicator is often biased. Confirmed use is defined as when the evaluation tests, observes or confirms a product or service was used in some way (e.g. via observation). Primary qualitative research studies: we included outcomes exploring the determinants of service utilisation of the MISP and its components.

Study types and design Primary quantitative research studies: Study designs including randomised controlled trials, non-randomised controlled trials, controlled before-after studies, controlled interrupted time series studies, economic studies (cost-effectiveness analysis, cost-utility analysis, cost-benefit analysis, economic modelling) of public health which the outcome is measured before and after the intervention or an intervention is studied against another intervention with baseline or control group. Primary qualitative research studies: Study designs including focus group discussions, in-depth interviews and structured observations, limiting studies to those with an observable contrast of time and/or a control group.
Studies which do not evaluate utilisation of SRH interventions extraction fields including study author and year, setting, target population, crisis type, crisis stage, study design and methods, research setting, study outcomes and intervention descriptions. Two researchers independently conducted screening of titles and abstracts, followed by data extraction from full-text review. Discrepancies in data extraction were resolved by arbitration by an additional independent researcher.

We used a narrative synthesis approach due to the heterogeneity of study outcomes, interventions and methods. ${ }^{9}$ The narrative synthesis approach comprises researchers moving in an iterative manner between the following four elements: (i) developing a theory of how the intervention works, why and for whom; (ii) developing a preliminary synthesis of findings of included studies; (iii) exploring relationships in the data and (iv) assessing the robustness of the synthesis. Findings were synthesised by main SRH outcomes, ie, family planning; abortion; prevention, treatment, care for STIs including HIV/AIDS; maternal and newborn health, including 
obstetric care; gender-based violence and studies with cross-cutting SRH themes. These themes were developed iteratively after conducting thematic analysis of the stated aims and primary reported health outcome of interest of the studies, which was done by manually coding the papers and reviewing data extracted to the Excel database.

The quality of reporting in the included studies was assessed using the Strengthening the Reporting of Observational Studies in Epidemiology (STROBE) and Critical Appraisal Skills Programme (CASP) checklists, ${ }^{10}{ }^{11}$ which are commonly used for reporting quality of observational and qualitative studies, respectively. The STROBE checklist includes measures on reporting of participant selection, variables, data sources, bias, descriptive and outcome data, interpretation and generalisability, among others. The CASP checklist includes measures on reporting of clear aims, use of appropriate methodology, research design and participant selection, adequate consideration of the relationship between the researcher and participants, rigour of data analysis, among others. To further explore the quality of reporting, we awarded papers one point for reporting each of the items on the relevant checklist. When totalled, these points formed the numerator of a proportional score, with the denominator being the total number of possible relevant checklist items. This denominator varied slightly by study type, that is, 22 points for the STROBE checklist and 10 points for the CASP checklist. NSS, SA and JS conducted quality assessment; each independently evaluated the quality of all included studies and discussed each discrepancy until consensus was reached. The study team determined a priori that papers were considering low reporting quality if the scoring was $<33 \%$, moderate quality if $34 \%-66 \%$ and high quality if $>67 \%$. These quality thresholds have been used in a previous systematic review on SRH in humanitarian crises settings. ${ }^{5}$

\section{Role of the funding source}

The funder of the study had no role in study design, data collection, data analysis or data interpretation. The corresponding author had full access to all data in the study and had final responsibility for the decision to submit for publication.

\section{Ethics}

Ethics approval was not applicable.

\section{RESULTS}

We screened titles and abstracts of 2404 citations, 2402 from literature searches and 2 from screening reference lists of other relevant systematic reviews. After full-text screening, 23 studies met our inclusion criteria and were selected for final review (figure 1). We identified studies focusing on family planning, HIV/STIs, maternal and newborn health and gender-based violence. We found no studies with interventions specifically focusing on PMTCT of HIV, vaginal injuries and fistula, post-abortion care, safe abortion, prevention of sexual violence and comprehensive clinical management of rape.

The 23 included studies were conducted in 16 countries with populations affected by armed conflict, natural disaster or both (table 2). Between 1997 and 2009, nine papers were published that met our inclusion criteria. Between 2010 and 2015, 14 more papers were found. Of these 23 studies, 22 used cross-sectional, pre-post and cohort study designs. One paper included in this review used qualitative research methods, specifically focus group discussions.

\section{Study quality}

Of the 23 studies, $22(95.7 \%)$ were assessed for quality of reporting using the STROBE checklist and the only qualitative study (4.3\%) was assessed using the CASP checklist for qualitative studies. Of the observational studies, four $(17.4 \%)$ were found to be of low quality, ${ }^{12-15}$ nine $(39.1 \%)$ were of moderate quality ${ }^{16-24}$ and the remaining nine $(39.1 \%)$ were of high quality. ${ }^{25-33}$ The included qualitative study was also deemed to be high quality. ${ }^{34}$

Overall, there were common areas in which the included studies in this review provided low quality reporting. First, all the moderate quality and low quality quantitative studies stated changes in utilisation outcomes, but statistical associations between the intervention and the outcome were not given. Second, it was also not clear whether relevant confounders and biases were considered during the design of the study and analysis of the data.

The following section synthesises findings on the utilisation of SRH interventions in humanitarian crises settings by main SRH outcomes.

\section{Family planning}

We identified six studies whose primary aim was to evaluate an intervention to improve the use of family planning services in humanitarian crises settings. A pre-post, multi-site study of moderate quality conducted in Chad, Democratic Republic of Congo (DRC), Mali, Djibouti and Pakistan evaluated the impact of staff training, facility supervision, supply of contraceptives as well as community awareness and mobilisation activities on the contraceptive uptake among women of reproductive age. The study found a general increase in new users of modern family planning methods over time, notably for new users choosing long-acting and reversible contraceptives (78\% in the DRC, $72 \%$ in Chad, $51 \%$ in Mali, $29 \%$ in Pakistan). ${ }^{16}$ A study of high quality assessing a home-based counselling and awareness programme for internally displaced women in Sudan led to an increase in the use of modern family planning methods (adjusted OR (aOR) $2.8,95 \%$ CI 2.0 to 4.1$).{ }^{27}$ Another study of high quality assessed a refugee-led reproductive health group operating across 48 Guinean refugee camps. ${ }^{25}$ The intervention recruited refugee nurses and midwives to local health facilities, trained lay women to provide health education and contraception and to facilitate referrals. Individuals 


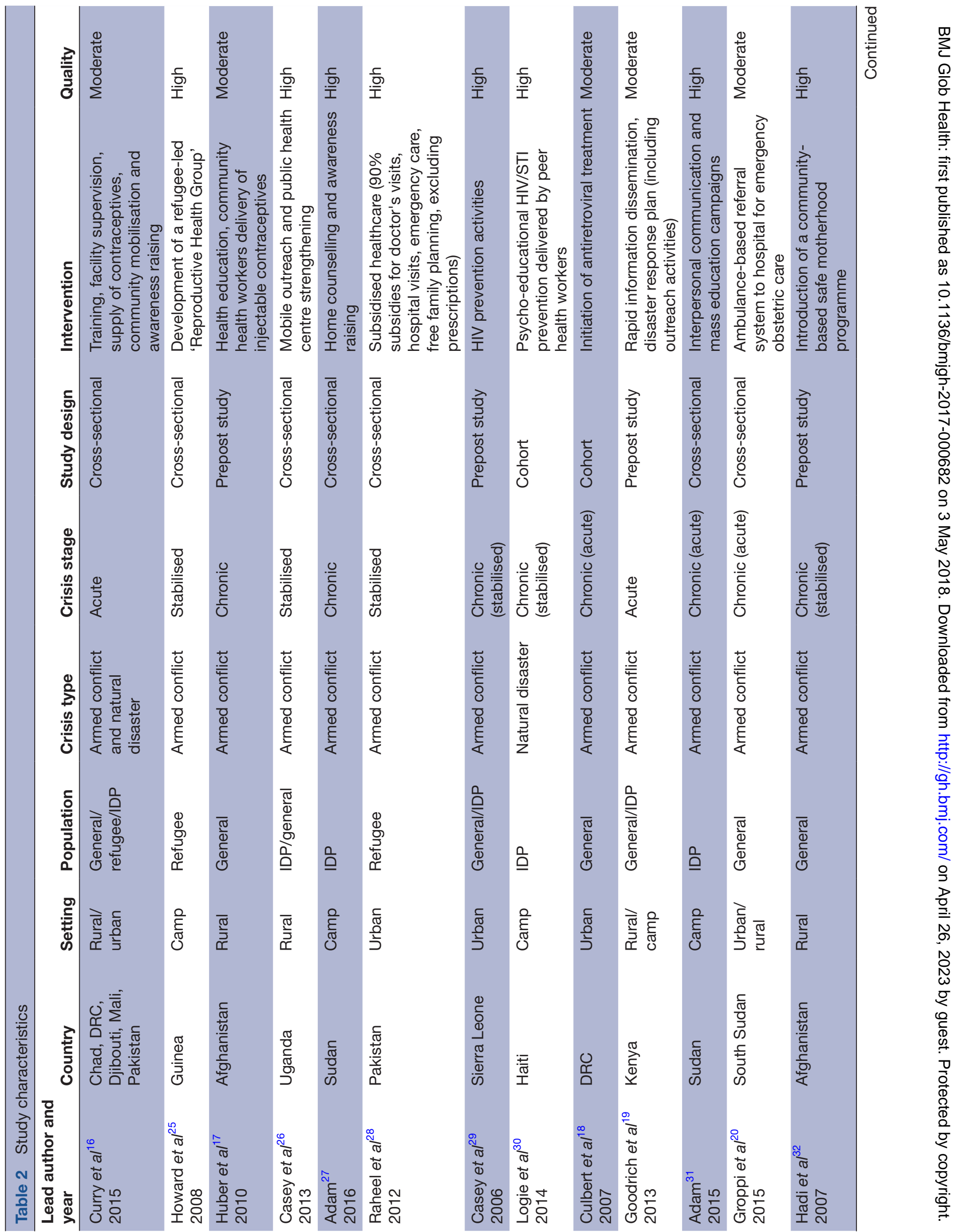




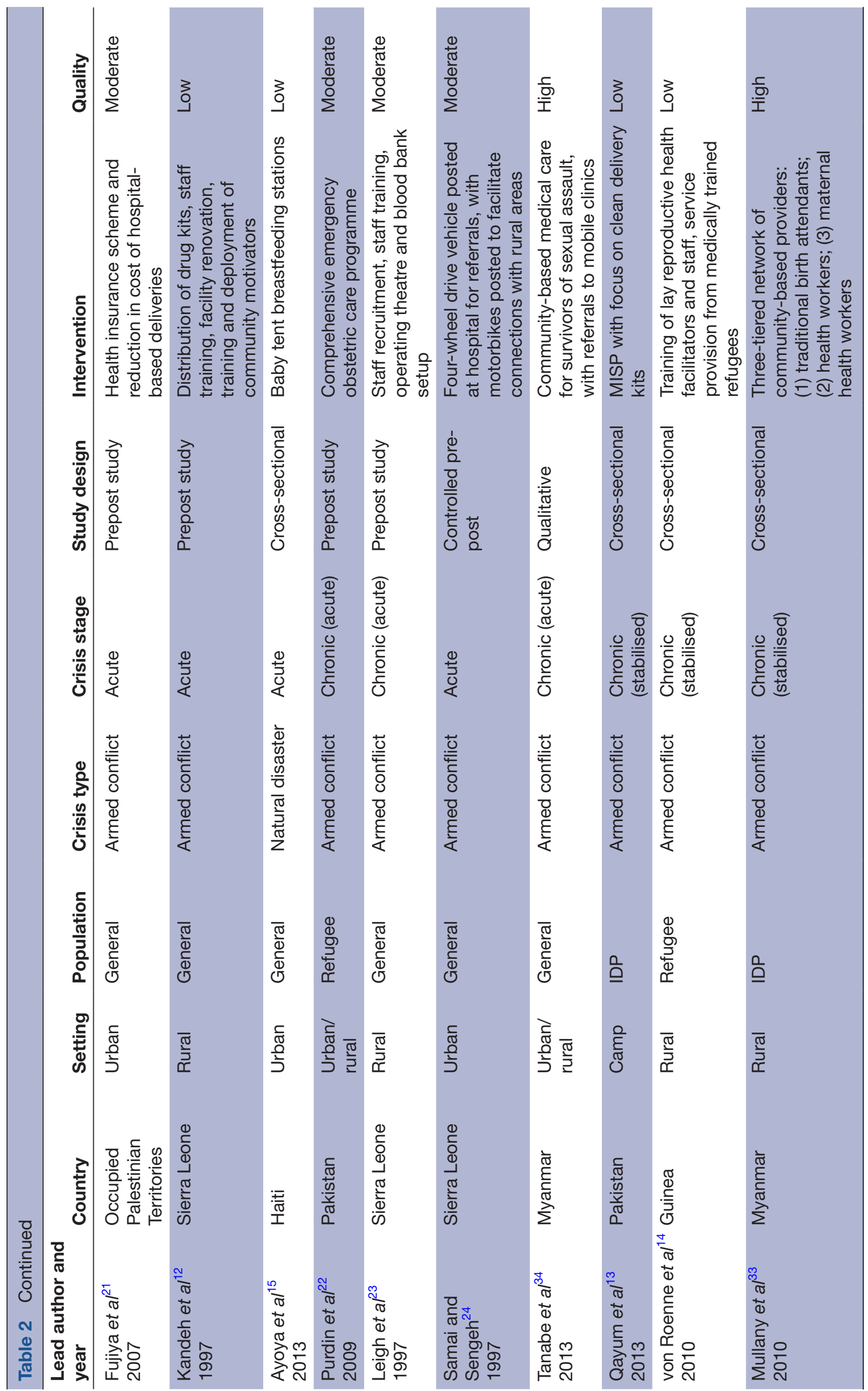


who reported the reproductive health group facilitators as their primary source of information increased their use of contraception (aOR 1.3, 95\% CI 0.7 to 4.2, non-significant). In Uganda, a high quality study assessing a mobile outreach intervention and public health strengthening programme led to an increase in the number of women who reported ever using a family planning method (aOR $2.23,95 \% \mathrm{CI} 1.7$ to $2.92, \mathrm{P}<0.001$ ), and a reduction in the unmet need for family planning from $52.1 \%$ to $35.7 \%$ (aOR $0.47,95 \%$ CI 0.37 to $0.6, \mathrm{P}<0.001$ ).$^{26}$ A study of moderate quality in rural Afghanistan assessed the impact of health education and the delivery of injectable contraceptives by community health workers (CHWs). ${ }^{17}$ Over an 8-month period in 2005-2006, contraceptive use increased by $24 \%-27 \%$ across three sites. ${ }^{17}$ Another high quality study in Pakistan assessed the provision of subsidised healthcare to refugees, reporting use of contraceptives in the subsidised group (54\%) was more than double the use reported in the non-subsidised group $(25 \%)(\mathrm{P}<0.001)$; and reporting that the non-subsidised group was more likely to use the pill $(40.7 \%)$, whereas the subsidised group was more likely to have tubal ligation $(36.7 \%)(\mathrm{P}<0.001) .{ }^{28}$

\section{Prevention, treatment and care for HIV/AIDS and STIS}

We identified four studies that focused on improving the utilisation of prevention, treatment and care services for HIV/AIDS and STIs. Two high quality studies evaluated the impact of HIV prevention interventions on condom use. Casey et al. assessed HIV prevention education activities and their subsequent effect on contraceptive use among adolescents aged 15-24 years using a pre-post study design. ${ }^{29}$ The study found that at baseline, approximately $15.6 \%$ of female and male adolescents reported using a condom the last time they had sex, while this proportion increased to $46.2 \%(\mathrm{P}<0.01)$ and $37.1 \%(\mathrm{P}<0.01)$, respectively after the intervention. Additionally, $24.8 \%$ of adolescents reported ever having used a condom at baseline, while this increased to $63.6 \%$ post-intervention. ${ }^{29}$ A cohort study conducted in Haiti after the 2012 earthquake aimed to evaluate a psycho-educational HIV/ STI prevention programme that was delivered by female peer health workers. ${ }^{30}$ The programme consisted of an HIV/STI educational video-based session, and a 6-week psycho-educational programme with weekly group meetings. A statistically significant change in condom use was identified post-intervention, after adjusting for confounders (OR $4.05,95 \%$ CI 1.86 to $8.83, \mathrm{P}<0.05){ }^{30}$

Two moderate quality studies specifically focused on assessing the utilisation of antiretroviral therapy (ART). The first, a pre-post study conducted in a camp setting during postelection violence in Kenya, evaluated a disaster response plan that included rapid information dissemination through the media, HIV outreach activities and a medical record system helping to track missed appointments among HIV-positive patients. ${ }^{19}$ Using clinic attendance as the primary outcome, the study reported that the majority of clinics $(60.0 \%, \mathrm{n}=12)$ had more visits during their opening week, and that there was an overall increase in patient volume by $105 \%$ compared with the baseline. The number of unscheduled visits also increased from $27.2 \%$ pre-intervention to $42.4 \%$ three months after clinics were opened. Additionally, there was a decline in the number of missed patient visits, although an exact proportion of decrease was not reported. The second was a cohort study conducted in the DRC to evaluate an ART intervention that included establishing voluntary counselling and testing sites in addition to HIV clinics. ${ }^{18}$ During the four years of the programme, 110076 people received voluntary counselling and testing, and of these people, $19 \%$ tested HIV positive. Ninety-four per cent of those testing positive received care in HIV clinics. By the fourth year of the programme, $26 \%$ of patients had commenced ART. Good ART adherence was achieved by $99 \%$ of patients.

\section{Maternal and newborn health including obstetric care}

Ten studies were identified whose primary aim was to improve maternal, obstetric and newborn health services. Of these, three studies specifically focused on increasing service utilisation of maternal healthcare in humanitarian crises settings. ${ }^{20} 3132$ Adam conducted a high quality cross-sectional study to evaluate an intervention aiming to increase antenatal care (ANC) attendance among recently pregnant women aged $15-48$ years using interpersonal communication and mass education campaigns over a 2-year time period. ${ }^{31}$ These education campaigns were found to increase the likelihood of a pregnant woman attending at least three ANC visits (OR 8.8, 95\% CI 6.4 to 12), and having at least three tetanus doses (OR 2.5, 95\% CI 1.9 to 3.3). There were also increases in the likelihood of the woman having a facility-based delivery (OR 5.4, 95\% CI 4.0 to $7.4, \mathrm{P}<0.001)$ and attending at least one postnatal care visit (OR 5.5, 95\% CI 4.0 to $7.7, \mathrm{P}<0.001)$. In a high quality study, Hadi et al. examined the effect of introducing a community-based safe motherhood programme in Afghanistan on utilisation of ANC services and institutional delivery. ${ }^{32}$ In both instances, there was statistical evidence of an increase in service utilisation compared to baseline. There was a $53.9 \%$ increase $(\mathrm{P}<0.01)$ in ANC services received from 2004 to 2006 , and a $23.9 \%$ increase $(P<0.01)$ in facility-based deliveries over the 2-year time period of the study. ${ }^{32}$

Fujiya $e t$ al. used a moderate quality pre-post study to evaluate the effect of a health insurance scheme and a reduction in the cost of hospital-based deliveries in the Occupied Palestinian Territories during an Israeli military invasion. ${ }^{21}$ There was a $9.1 \%$ increase in live births at government hospitals when health insurance was provided to pregnant women. There was also a consistent increase in the number of births at the study hospital once the price was reduced for facility-based deliveries.

Additionally, we identified two studies that reviewed interventions aiming to reduce the unmet needs for emergency obstetric care (EmOC). In a low quality 
pre-post study, Kandeh et al. evaluated the impact of an intervention in Sierra Leone between 1992 and 1995 that included the distribution of drug commodities to health units, staff training, facility renovation and the use of community motivators. ${ }^{12}$ An analysis of met needs during implementation showed that health facilities were seeing $43 \%$ of the total expected number of women with complications in the area during the first study period, prior to most interventions, with this figure rising to $59 \%$ after full implementation of the intervention. A moderate quality pre-post study conducted in Pakistan also evaluated the effect of a comprehensive EmOC programme on facility-based deliveries. ${ }^{23}$ During the intervention period, the proportion of births in an EmOC facility increased from $4.8 \%$ at baseline to $67.2 \%$ post-intervention.

One pre-post study of moderate quality was conducted in Sierra Leone between 1990 and 1995 to assess an intervention with the specific aim of increasing facility utilisation by improving the infrastructure of a rural health facility, and by providing training. ${ }^{12}$ Additional staff were recruited and trained in obstetric care, and an operating theatre and blood bank were established. The study found that maternal admissions more than doubled in the first year of the intervention when only the first medical officer with obstetric skills was posted. There was a fourfold increase of maternal admissions of those with obstetric complications. ${ }^{12}$

Two moderate quality studies were identified that evaluated vehicle-based interventions to increase the use of obstetric services in emergencies. Samai et al. used a controlled pre-post study to evaluate the impact of a fourwheel vehicle that was posted to a government hospital in Sierra Leone, with motorbikes to provide additional linkages between primary health units and the vehicle. ${ }^{24}$ The intervention also included community education activities, provision of drugs and improvement to the infrastructure of the hospital. A radio communication referral system was also established to improve communication between the hospital and rural areas. The study found that utilisation of EmOC services doubled, but that this improvement could also be attributed to improved community awareness of obstetric emergencies and improved care at the hospital. ${ }^{24}$ Groppi et al. used a cross-sectional study to determine the impact of an ambulance-based referral system to a hospital for EmOC services in South Sudan by collecting data on facility deliveries prospectively. ${ }^{20}$ One year post-intervention, $13.3 \%$ of the expected deliveries in the hospital's catchment area took place at the facility. The study found that $22.0 \%$ of all ambulance referrals to the hospital that year were for women with major obstetric complications.

Lastly, one low quality study assessed an intervention aiming to improve the use of neonatal and child care services by assessing the utilisation of baby tent breastfeeding stations in Haiti by breastfeeding women after a natural disaster, using a cross-sectional study design. ${ }^{15}$ The study reported that $54 \%$ of the infants enrolled at a breastfeeding station were aged $<6$ months, and that
$70 \%$ of these infants were exclusively breastfed. The remaining infants were reported to be receiving 'mixed feeding', but $10 \%$ moved to exclusive breast feeding by the end of their participation in the programme. ${ }^{15}$

\section{Gender-based violence}

One high quality qualitative study was identified whose primary aim was to provide data on the feasibility of providing community-based medical care for sexual assault survivors as part of a larger process evaluation of the intervention. ${ }^{34}$ Conducted in Myanmar in an armed conflict setting, this study used focus group discussions with community members, traditional birth attendants and CHWs to understand the utilisation of a community-based medical care package for survivors of sexual assault delivered at the community level. This package was adapted from the 2004 WHO's Clinical Management of Rape Survivors facility-based protocol so that it could be delivered by CHWs. The study found that some of the perceived barriers and challenges for sexual assault survivors in accessing and using services are shyness, shame, fear of others' opinions and fear that they may be denied help. It was also suggested that generally, the community needed to feel more comfortable in seeking care from CHWs. ${ }^{34}$

\section{Interventions targeting outcomes across multiple SRH domains}

In total, three studies were identified that evaluated interventions that focused on more than one of the SRH domains mentioned above. One low quality study used a household cross-sectional survey to evaluate whether internally displaced people within a camp setting in Pakistan were able to access the MISP, and implemented services with a special focus on the provision of clean delivery kits. ${ }^{13}$ Findings report $23 \%$ of women had problems in accessing ANC and delivery services at the health facilities, and $87 \%$ of women answered that they did not receive follow-up visits or check-ups during their pregnancy by a health worker. Over three-quarters $(78 \%)$ of the women surveyed also reported they had not received clean delivery kits. ${ }^{13}$

The remaining two articles were both cross-sectional studies and evaluated interventions providing family planning and maternal health services. One low quality study evaluated an intervention in which lay reproductive health facilitators and staff recruited from the refugee population in Guinea were trained in the provision of family planning and maternal health services. ${ }^{14}$ The study found that the contraceptive prevalence rate among refugees in the intervention's catchment area was $25 \%$ higher than the national country average, and that ANC coverage was $54 \%$ in its catchment area post-intervention, which was higher than the estimated ANC coverage average (ranging from $11 \%$ to $42 \%$ ) among the refugee population in Guinea prior to implementation. Lastly, a high quality study evaluated the impact of a three-tiered network of community-based providers in 
Myanmar, which included traditional birth attendants, health workers and maternal health workers. ${ }^{33}$ With this intervention, the use of modern contraception methods increased from $23.9 \%$ to $45.0 \%$ (prevalence rate ratio $(\mathrm{PRR})=1.9,95 \%$ CI 1.6 to 2.2$)$. The number of women receiving at least one ANC visit or more increased from $39.3 \%$ to $71.8 \%$ (PRR $=1.8,95 \%$ CI 1.6 to 2.0 ). Deliveries were also 9.6 times more likely to be attended by a provider trained in EmOC post-intervention ( $\mathrm{PRR}=9.6$, 95\% CI 7.2 to 12.6$).{ }^{33}$

\section{DISCUSSION}

This is the first systematic review to assess the utilisation of SRH interventions, including the MISP, in humanitarian crises. Of the 2404 citations screened, the review identified 23 studies since 1980 assessing the utilisation of SRH services in humanitarian crises that met the inclusion criteria. Interventions resulting in increased SRH service utilisation included interpersonal and peer-led education and mass media campaigns, focused community-based programming and tiered community health service provision to improve reproductive and maternal health services. Lower quality evidence was found to support general health system strengthening efforts combined with the use of community motivators, birth preparedness interventions and the training of lay SRH facilitators to provide family planning and ANC. We identified no studies that evaluated the utilisation of interventions focused on PMTCT, STI treatment and prevention excluding HIV/AIDS, vaginal injuries and fistula, post-abortion care, safe abortion, prevention of sexual violence, comprehensive clinical management of rape or of the MISP as a comprehensive package of interventions.

The quality of evidence identified in this review was variable, with particularly weak study design and most studies graded as high and moderate quality, since study design was only one component in the STROBE and CASP checklists used to assess study quality. Just over one-third $(36.7 \%)$ of the studies $(n=7)$ used quasi-experimental study designs, which provided some statistical measure of difference between intervention and outcome. This also meant that, where appropriate, there was insufficient adjustment for potential confounders. Evidence on attribution is particularly weak, with the vast majority of studies using a cross-sectional or pre-post study design, with no control group. Qualitative studies have the potential to contribute rich perspectives from study populations on service utilisation, but we found only one study using this design, and no study using mixed methods to assess SRH service utilisation in humanitarian crises. Overall, only $39.1 \%$ of the studies $(n=9)$ selected were graded as high quality, $39.1 \%$ as moderate quality $(\mathrm{n}=9)$ and $17.4 \%$ as low quality $(n=4)$. There was limited use of stratification, for example, by gender or age, and so we were not able to capture potentially differing health utilisation outcomes in more vulnerable group such as adolescent girls. Use of STROBE and CASP tools identified fundamental methodological issues with most of the available evidence including: lack of appropriate study design; absence of reporting on sampling methods; lack of control groups and randomisation procedures; limited appreciation of clear exposures and confounders and an inadequate handling of bias. Statistical analysis was reported in only five studies $(21.7 \%)$, signalling lack of robust research in this field. A recent systematic review on public health interventions in humanitarian crises found a greater share of studies on communicable diseases and nutrition in humanitarian crises, ${ }^{4}$ suggesting that more rigorous SRH research is possible in these settings. We did not identify any cost-effectiveness studies, which is of concern as it highlights gaps in the economic data required by policymakers and programme implementers to deliver and scale up SRH services.

We found a similar level of evidence and quality of studies measuring SRH utilisation compared with previous systematic reviews evaluating SRH interventions in humanitarian crises, ${ }^{15}$ signalling slower progress in advancing the evidence base in this field. It was not possible to synthesise findings by setting (e.g. camps, urban areas) or population type (e.g. adolescents), given the paucity of identified studies. Casey $e t a l .^{29}$ found that adolescents access health services in different ways than the general population and have different concerns (e.g. privacy) and health needs, signalling the importance of conducting studies that specifically target this population and assess their utilisation of SRH services. ${ }^{29}$ In addition to research on delivering and scaling up SRH services to different target populations, we should also consider implementation research in different humanitarian crises settings. For example, the private sector and informal providers may play a larger role in service delivery in protracted humanitarian crises than in acute settings where non-governmental organisations may be delivering most of the services. These evidence gaps point to the need for robust and timely research on the mechanisms through which SRH interventions can increase utilisation by key populations across a variety of humanitarian crises.

This review has a number of limitations. Although we included qualitative and quantitative studies in our study design criteria, other narrow inclusion criteria may have led to the exclusion of some peer-reviewed literature. Additionally, our language inclusion criteria, i.e. only studies published in English or French, imposed by the capacity of the study team, may have limited the numbers of citations returned by our search. We also note that the STROBE checklist only assesses reporting of studies, rather than the actual design, sampling/recruitment and the appropriateness of analysis methods. Study outcomes, intervention types and methods varied widely across the 23 included studies, which did not allow us to conduct a meta-analysis. We also acknowledge that the varying geography, social and cultural diversity of study settings and populations included in our review would have an impact on the utilisation of SRH interventions in these settings, 
and might lead to limited external validity for some SRH interventions. However, no studies reported measuring these distal determinants, so we were unable to assess their effect on the utilisation of SRH interventions.

\section{CONCLUSIONS}

The review found some evidence to support increased utilisation of SRH services through peer-led and interpersonal education and mass media campaigns, community-based programming and a three-tiered network of community-based providers for reproductive and maternal health services. However, these results should be interpreted cautiously due to limited use of statistical data and less robust study design. Rigorous and timely research, as well as high quality evidence to support the use of different intervention designs, is needed to identify evidence-based interventions to increase the utilisation of SRH services by key populations across a range of diverse humanitarian crises settings.

Twitter neha_s_singh

Acknowledgements The authors would like to thank Maria Rodriguez, Loulou Kobeissi, Adam Poulter, Robyn Drysdale, Rajrattan Rokhande and Sara Nam for reviewing the study protocol.

Contributors NSS and KB led the study. NSS, SA and JS participated in data searching and data analysis. NSS led the writing up of the paper with SA. RK and LS participated in development of the study protocol and reviewed drafts of the paper. All authors contributed to the paper.

Funding This work was funded with support from the Department of Reproductive Health and Research, WHO.

Disclaimer The authors alone are responsible for the views expressed in this publication, and they do not necessarily represent the decisions, policy or views of WHO.

Competing interests All authors declare support from WHO for the submitted work; no financial relationships with any organisations that might have an interest in the submitted work in the previous 3 years, no other relationships or activities that could appear to have influenced the submitted work.

Patient consent Not required.

Provenance and peer review Not commissioned; externally peer reviewed.

Data sharing statement No additional data are available.

Open Access This is an Open Access article distributed in accordance with the terms of the Creative Commons Attribution (CC BY 4.0) license, which permits others to distribute, remix, adapt and build upon this work, for commercial use, provided the original work is properly cited. See: http://creativecommons.org/ licenses/by/4.0/

(C) Article author(s) (or their employer(s) unless otherwise stated in the text of the article) 2018. All rights reserved. No commercial use is permitted unless otherwise expressly granted.

\section{REFERENCES}

1. Casey SE. Evaluations of reproductive health programs in humanitarian settings: a systematic review. Confl Health 2015;9:S1.

2. State of world population. New York: United Nations Population Fund, 2015.

3. Lisam S. Minimum initial service package (MISP) for sexual and reproductive health in disasters. J Evid Based Med 2014;7:245-8.

4. Blanchet K, Ramesh A, Frison S, et al. Evidence on public health interventions in humanitarian crises. Lancet 2017;390:2287-96.

5. Warren E, Post N, Hossain M, et al. Systematic review of the evidence on the effectiveness of sexual and reproductive health interventions in humanitarian crises. BMJ Open 2015;5:e008226.
6. Moher D, Liberati A, Tetzlaff J, et al. Preferred Reporting Items for Systematic Reviews and Meta-Analyses: The PRISMA Statement. PLoS Med 2009;6:e1000097.

7. Report of the international conference on population and development. 1994. International Conference on Population and Development. New York:United Nations.

8. World Health Organization. Reproductive health strategy to accelerate progress towards the attainment of international development goals and targets: Global strategy adopted by the 57th World Health Assembly. 2004 http://www.who.int/ reproductivehealth/publications/general/RHR_04_8/en/

9. Petticrew M, Rehfuess E, Noyes J, et al. Synthesizing evidence on complex interventions: how meta-analytical, qualitative, and mixed-method approaches can contribute. J Clin Epidemiol 2013;66:1230-43.

10. Knottnerus A, Tugwell P. STROBE-A checklist to strengthen the reporting of observational studies in epidemiology. J Clin Epidemiol 2008;61:323.

11. Critical Appraisal Skills Programme (CASP). CASP qualitative checklist. $2013 \mathrm{http}: / /$ mediawixcom/ugd/ dded87_29c5b002d99342f788c6ac670e49f274pdf

12. Kandeh HBS, Leigh B, Kanu MS, et al. Community motivators promote use of emergency obstetric services in rural Sierra Leone. Inter J Gynecol Obst 1997;59(Suppl 2):S209-18.

13. Qayum M, Arooj H, Mohmand S. Minimum Initial Service Package (MISP) access to displaced people of Pakistan based on sphere standards and indicators. J Pak Med Assoc 2013;63:1027-30.

14. von Roenne A, von Roenne F, Kollie S, et al. Reproductive health services for refugees by refugees: an example from Guinea. Disasters 2010;34:16-29.

15. Ayoya MA, Golden K, Ngnie-Teta I, et al. Protecting and improving breastfeeding practices during a major emergency: lessons learnt from the baby tents in Haiti. Bull World Health Organ 2013;91:612-7.

16. Curry DW, Rattan J, Huang S, et al. Delivering high-quality family planning services in crisis-affected settings II: results. Glob Health Sci Pract 2015;3:25-33.

17. Huber D, Saeedi N, Samadi AK. Achieving success with family planning in rural Afghanistan. Bull World Health Organ 2010;88:227-31.

18. Culbert $H, T u D, O^{\prime} B r i e n ~ D P$, et al. HIV treatment in a conflict setting: outcomes and experiences from Bukavu, Democratic Republic of the Congo. PLoS Med 2007;4:e129.

19. Goodrich S, Ndege S, Kimaiyo S, et al. Delivery of HIV care during the 2007 post-election crisis in Kenya: a case study analyzing the response of the Academic Model Providing Access to Healthcare (AMPATH) program. Confl Health 2013;7:25.

20. Groppi L, Somigliana E, Pisani V, et al. A hospital-centered approach to improve emergency obstetric care in South Sudan. Int J Gynaecol Obstet 2015;128:58-61.

21. Fujiya R, Jimba M, Giacaman R, et al. The influence of economic factors on the location of birth among Palestinian women in Bethlehem during the second Palestinian uprising. Trop Doct 2007;37:13-17.

22. Purdin $\mathrm{S}$, Khan $\mathrm{T}$, Saucier R. Reducing maternal mortality among Afghan refugees in Pakistan. Int J Gynaecol Obstet 2009;105:82-5.

23. Leigh B, Kandeh HB, Kanu MS, et al. Improving emergency obstetric care at a district hospital, Makeni, Sierra Leone. The Freetown/ Makeni PMM Team. Int J Gynaecol Obstet 1997;59(Suppl 2):S55-65.

24. Samai $O$, Sengeh P. Facilitating emergency obstetric care through transportation and communication, Bo, Sierra Leone. The Bo PMM Team. Int J Gynaecol Obstet 1997;59(Suppl 2):S157-64.

25. Howard N, Kollie S, Souare Y, et al. Reproductive health services for refugees by refugees in Guinea I: family planning. Confl Health 2008;2:12.

26. Casey SE, McNab SE, Tanton C, et al. Availability of long-acting and permanent family-planning methods leads to increase in use in conflict-affected northern Uganda: evidence from crosssectional baseline and endline cluster surveys. Glob Public Health 2013;8:284-97.

27. Adam IF. Evidence from cluster surveys on the association between home-based counseling and use of family planning in conflictaffected Darfur. Int J Gynaecol Obstet 2016;133:221-5.

28. Raheel H, Karim MS, Saleem S, et al. Knowledge, attitudes and practices of contraception among Afghan refugee women in Pakistan: a cross-sectional study. PLoS One 2012;7:e48760.

29. Casey SE, Larsen MM, McGinn T, et al. Changes in HIV/AIDS/STI knowledge, attitudes, and behaviours among the youth in Port Loko, Sierra Leone. Glob Public Health 2006;1:249-63.

30. Logie $\mathrm{CH}$, Daniel C, Newman PA, et al. A psycho-educational HIV/STI prevention intervention for internally displaced women in 
Leogane, Haiti: results from a non-randomized cohort pilot study. PLoS One 2014;9:e89836.

31. Adam IF. The influence of maternal health education on the place of delivery in conflict settings of Darfur, Sudan. Confl Health 2015;9.

32. Hadi A, Rahman T, Khuram D, et al. Raising institutional delivery in war-torn communities: experience of BRAC in Afghanistan. Asia Pacific Family Medicine 2007;6.
33. Mullany LC, Lee TJ, Yone L, et al. Impact of community-based maternal health workers on coverage of essential maternal health interventions among internally displaced communities in eastern Burma: the MOM project. PLoS Med 2010;7:e1000317.

34. Tanabe M, Robinson K, Lee Cl, et al. Piloting community-based medical care for survivors of sexual assault in conflict-affected Karen State of eastern Burma. Confl Health 2013;7:12. 